\title{
PRODUCCIÓN DE LA MICROALGA Nannochloropsis oculata (Droop) Hibberd EN MEDIOS ENRIQUECIDOS CON ENSILADO BIOLÓGICO DE PESCADO
}

\section{PRODUCTION OF THE MICROALGAE Nannochloropsis oculata (Droop) Hibberd ON MEDIA ENRICHED WITH BIOLOGICAL FISH ENSILAGE}

\author{
Heidi Sánchez Torres ${ }^{1}$, Juan Juscamaita Morales ${ }^{1}$, Jessie Vargas Cárdenas ${ }^{2}$ y Ricardo Oliveros Ramos ${ }^{3}$
}

\begin{abstract}
Resumen
Los residuos pesqueros se producen en grandes volúmenes en el Perú, generando problemas de contaminación, sin embargo, estos pueden ser un favorable sustrato para aplicaciones biotecnológicas. El ensilado biológico de pescado puede suplir a los medios de cultivo masivo basados en fertilizantes agrícolas para la producción de microalgas. Se cultivó a Nannochloropsis oculata en lote durante 12 días hasta alcanzar la fase estacionaria, con temperatura e iluminación constantes las 24 horas del día. Se compararon siete diferentes medios de cultivo (T1, Guillard F/2, T2, Yashima y T3-T6, cuatro medios basados en ensilado de pescado, y T7 harina de pescado). Los medios basados en ensilado de pescado fueron dos con diferentes ensilados (hidrolizados y no-hidrolizado) y dos por enriquecimiento del medio Yashima con ensilado nohidrolizado en diferentes concentraciones. El mejor de los tratamientos experimentales fue el del medio basado en ensilado de pescado no-hidrolizado (T6), no encontrándose diferencias significativas en la productividad máxima de este cultivo comparado con la del medio Yashima (1.65 g.L $\left.\mathrm{L}^{-1} \cdot \mathrm{d}^{-1}\right)$. La producción mensual estimada utilizando este medio fue de $41.5 \mathrm{~g} \cdot \mathrm{mes}^{-1} \cdot \mathrm{L}^{-1}$. Se evaluó la calidad nutricional de la biomasa de $N$. oculata obtenida como alimento vivo para el rotífero Brachionus plicatilis y se encontró que el tratamiento T6 obtuvo un mayor índice de fecundidad, en comparación al tratamiento T2, lo que es un indicador de buena calidad nutricional. El medio basado en ensilado de pescado mostró una alta producción, bajo costo y buena calidad nutricional para la producción de microalgas.
\end{abstract}

Palabras clave: Acuicultura, medios orgánicos, residuos pesqueros, microalgas, Nannochloropsis, Brachionus

\begin{abstract}
Fishing residues are produced in large volumes in Peru, generating pollution problems, but conversely can be a favourable substrate for biotechnological applications. Biological fish ensilage can replace massive culture media based on agricultural fertilizers for microalgae production. Nannochloropsis oculata was grown in batch culture during 12 days until stationary phase, under constant temperature and an illumination of $24 \mathrm{~h} . \mathrm{d}^{-1}$. Seven different culture media (T1, Guillard F/2, T2, Yashima and T3-T7 d four culture media based on biological fish ensilage and T7 fish floor) were compared. Media based on biological fish ensilage were: two with different kinds of ensilages (hidrolized and non-hidrolized) and two with Yashima medium plus non-hidrolized ensilage at two different concentrations. The best experimental treatment was the medium based on non-hidrolized biological fish ensilage (T6), but non significant differences in maximum productivity were found compared with culture on Yashima medium (1.65 g.L $\left.\mathrm{L}^{-1} . \mathrm{d}^{-1}\right)$. Monthly production using this medium was estimated in 41.5 g.month ${ }^{-1} \cdot \mathrm{L}^{-1} . N$. oculata biomass nutritional quality as live food for Brachionus plicatilis rotifer was evaluated and it was found that nonhidrolized ensilage treatment T6 had a greater fecundity index in comparison to Yashima treatment T2, which is an indicator of good nutritional quality. Biological fish ensilage medium showed a high production, low cost and good nutritional quality for the microalgae production.

Key words: Aquaculture, fishing residue, organic medium, microalgae, Nannochloropsis, Brachionus
\end{abstract}

\section{Introducción}

En la actualidad, la industria acuícola considera como un aspecto importante en el cultivo de organismos acuáticos, la calidad nutricional del alimento vivo (microalgas y zooplancton), ya que representan la base de la cadena alimenticia para la producción de peces, moluscos y crustáceos. La producción de alimento vivo (zooplancton y 
microalgas) a gran escala resulta dificultosa, debido fundamentalmente al costo que implica su producción, pues el sustrato para su cultivo debe cubrir todos los requerimientos nutricionales que permitan la composición química óptima del alimento vivo, pues un sustrato pobre en nutrientes esenciales puede causar el desarrollo anormal y muerte masiva de las especies en cultivo. Una alternativa a los medios de cultivo tradicionales ya estandarizados (puros y químicamente definidos, como el medio F/2 Guillard) son los sustratos orgánicos, mayormente subproductos de otras industrias, y en cuya composición se encuentra fuente de $\mathrm{C}, \mathrm{N}, \mathrm{P}$, microelementos y otros nutrientes. En la actualidad se utilizan diversas harinas (de pescado, soya, lombriz de tierra, girasol), gallinaza, extractos de excretas de vaca (a partir de estiércoles digeridos obtenidos en un biodigestor de recirculación aerobia), gas oil, melaza de caña, exudados gomosos de Acacia macracantha, extractos de macroalgas, bokashi y residuales pesqueros (De la Cruz \& Alfonso, 1975; Granados \& Bückle, 1983; Paniagua \& Bückle, 1983; Chinchayán, 1996; Leal et al., 2004; Veral et al., 2006).

En Perú, los residuos pesqueros se producen en grandes cantidades como consecuencia de la industria pesquera, que es la actividad extractiva de recursos renovables más importante del Perú. Los residuos como vísceras, cabezas, piel, huesos y colas, entre otros, se cuantifican alrededor del $50 \%$ de la materia prima, resultando finalmente en contaminantes al ser vertidos indiscriminadamente al medio ambiente, provocando problemas de contaminación y enfermedades. Sin embargo, estos residuos pueden ser aprovechados eficientemente si reciben un tratamiento para trasformarlos en fertilizantes. El tratamiento más conveniente para este tipo de residuos es el ensilaje biológico, el cual, mediante un proceso de fermentación acido-láctica, permite obtener un producto rico en nutrientes, de fácil elaboración y de bajo costo, con características idóneas para ser utilizado como fertilizante orgánico en acuicultura (Gonzáles \& Marín, 2005).

La microalga Nannochloropsis oculata (Droop) Hibberd es una microalga muy importante en acuicultura debido a su valor nutricional. Esta microalga pertenece a la clase Eustigmatophyceae, que agrupa a las especies que contienen la mayor cantidad de ácidos grasos poliinsaturados (PUFAs), especialmente ácido eicosapentaenoico (EPA), ácido araquidónico (ARA), docosahexaenoico (DHA) de gran importancia en la nutrición de animales marinos, especialmente en el crecimiento y desarrollo de larvas de peces, moluscos y crustáceos (Otero et al., 1997; Brown et al, 1999). La microalga $N$. oculata ha sido sugerida como un alimento preferible para el rotífero Brachionus plicatilis Mueller, el cual transfiere eficientemente los ácidos grasos poliinsaturados algales a las larvas de peces marinos (Sukenik, et al., 1993; Hoshida et al., 2005).

Vista la importancia de disminuir los costos en la producción del alimento vivo sin sacrificar la calidad nutricional y mitigar el impacto de los residuos emitidos por la industria pesquera en el ambiente, en este trabajo analizamos la posibilidad de utilizar el ensilado biológico de pescado como sustrato para el cultivo de la microalga $N$. oculata. Nuestro objetivo principal fue estudiar el efecto de las diversos tipos y concentraciones del ensilado biológico de pescado sobre el crecimiento de $N$. oculata y evaluar su calidad nutricional como alimento vivo para el rotífero $B$. plicatilis. Se busca también determinar la concentración óptima del ensilado biológico de pescado en el medio de cultivo para maximizar el rendimiento de biomasa de $N$. oculata, evaluar las diferencias en su productividad volumétrica por el efecto de las diferentes concentraciones del ensilado biológico de pescado como sustrato y la calidad nutricional de los cultivos de $N$. oculata mediante el análisis de las tasas de reproducción y fecundidad del rotífero B. plicatilis.

\section{Materiales y métodos}

Los cultivos se realizaron en el Laboratorio de Acuicultura, en el área de Alimento Vivo de la Facultad de Pesquería y en el Laboratorio de Biorremediación, de la Universidad Nacional Agraria La Molina (UNALM).

\section{Organismos}

Se utilizó una cepa de microalga Nannochloropsis oculata (Droop) Hibberd proveniente del Laboratorio de Microalgas del Instituto del Mar del Perú (IMARPE), que fue reaislada a partir de un cultivo masivo mediante la técnica de rayado en placa y el método de la pipeta capilar para obtener un cultivo axénico. La cepa de rotífero utilizada fue Brachionus plicatilis (línea S), procedente del stock de inóculos mantenidos en el Laboratorio de Alimento Vivo de la UNALM.

Medios y condiciones de cultivo

Se utilizó agua de mar natural con salinidad reducida de 40 \% a 20 \% con agua destilada, y se filtró posteriormente con un papel filtro de paso lento, para finalmente ser esterilizada por autoclavado. Se compararon 7 tratamientos. Los dos primeros medios de cultivo fueron usados como controles. El tratamiento 1 (T1, Guillard), consistió en agua de mar natural 20 \%o enriquecida con solución Guillard F/2 (composición final por litro: $75 \mathrm{mg} \mathrm{KNO}_{3}, 5.65 \mathrm{mg}$ $\mathrm{NaH}_{2} \mathrm{PO}_{4} .2 \mathrm{H}_{2} \mathrm{O}$, $4.360 \mathrm{mg}$ EDTA. $\mathrm{Na}_{2}, 3.150 \mathrm{mg}$ $\mathrm{FeCl}_{3} \cdot 6 \mathrm{H}_{2} \mathrm{O}, 0,010 \mathrm{mg} \mathrm{CuSO}_{4} \cdot 5 \mathrm{H}_{2} \mathrm{O}, 0,022 \mathrm{mg}$ $\mathrm{ZnSO}_{4} .7 \mathrm{H}_{2} \mathrm{O}$, 0,010 mg $\mathrm{CoCl}_{2} \cdot 6 \mathrm{H}_{2} \mathrm{O}, 0.180 \mathrm{mg}$ $\mathrm{MnCl}_{2} \cdot 4 \mathrm{H}_{2} \mathrm{O}, 0,006 \mathrm{mg} \mathrm{Na}_{2} \mathrm{MoO}_{4} \cdot 2 \mathrm{H}_{2} \mathrm{O}, 2 \mu \mathrm{g}$ cianocobalamina cristalina (B12), $0.100 \mathrm{mg}$ tiamina clorhídrica (B1), 0,001 mg biotina cristalina). El tratamiento 2 (T2, Yashima), en agua de mar natural 
20\%o enriquecida con solución Yashima (composición final por litro: $100 \mathrm{mg}$ sulfato de amonio para agricultura $21 \%$, 15mg superfosfato de calcio para la agricultura $21 \%, 15 \mathrm{mg}$ urea para la agricultura $21 \%$, Clewat 32 30-50 $\mu \mathrm{g})$. La solución Clewat 32 es usada como fuente de micro-nutrientes y está compuesta por $\mathrm{FeCl}_{2} 3.85$ g.L. ${ }^{-1}, \mathrm{ZnCl}_{2} 1.66$ g.L ${ }^{-1}, \mathrm{MnCl}_{2} 7.75$ g.L ${ }^{-1}$, $\mathrm{CoCl}_{2} 0.17$ g.L. ${ }^{-1}, \mathrm{CuSO}_{4}$ 0,07 g.L ${ }^{-1},\left(\mathrm{NH}_{4}\right) 6 \mathrm{Mo}_{7} \mathrm{O}_{24}$ 6.32 g.L. ${ }^{-1}, \mathrm{H}_{3} \mathrm{BO}_{3} 24.70$ g.L ${ }^{-1}$, EDTA. $\mathrm{Na}_{2}$ 0,05 g.L ${ }^{-1}$.

Para la preparación de los otros medios de cultivo, se obtuvo ensilado biológico de pescado del Laboratorio de Bioremediación de la Universidad Nacional Agraria La Molina, el cual fue preparado mediante ensilaje de residuos de pescado de consumo humano directo obtenidos de mercados minoristas por fermentación con bacterias ácido-lácticas con melaza como fuente de carbono. Se utilizaron dos tipos de ensilado biológico de pescado: hidrolizado $(\mathrm{H})$ y nohidrolizado (E) que diferían entre sí en el pretratamiento de los residuos. Para el ensilado hidrolizado, los residuos de pescado fueron previamente tratados con endoproteasas (hidrólisis enzimática). En un matraz estéril de $250 \mathrm{~mL}$ se filtraron por separado los fertilizantes Ensilado-E y Ensilado-H con la ayuda de un embudo estéril y papel Whatman $\mathrm{N}^{\circ} \mathrm{l}$. La solución resultante fue separada y conservada para enriquecer los medios de cultivo. El $\mathrm{pH}$ del ensilado-H (hidrolizado) fue de $3.6 \mathrm{y}$ el del ensilado E (no-hidrolizado) fue de 4.2. Los medios de los tratamientos 3-6 se seleccionaron a partir de los resultados de ensayos pre-experimentales con diversas concentraciones de ensilado, y consistieron en agua de mar natural 20 \%o enriquecida con: solución Yashima como en el tratamiento 2 y con la solución de Ensilado-H en una concentración de 1 mL.L ${ }^{-1}$ (T3), con solución Yashima como en el tratamiento 2 y con la solución de Ensilado-H en una concentración de $0.67 \mathrm{~mL} . \mathrm{L}^{-1}$ (T4) con solución de Ensilado-H en una concentración de 1 mL.L ${ }^{-1}$ (T5), con Ensilado-E en una concentración de $1 \mathrm{~mL} . \mathrm{L}^{-1}$ (T6). Finalmente, el tratamiento 7 (T7, harina de pescado) consistió en agua de mar natural 20 \%o enriquecida con harina de pescado $1000 \mathrm{ppm}$, los datos de este tratamiento se obtuvieron de Chinchayán (1996).

El cultivo fue en lote (batch) durante 12 días, tiempo en el cual todos los tratamientos alcanzaron la fase estacionaria del crecimiento. El cultivo del $N$. oculata se realizó a una temperatura $21^{\circ} \mathrm{C} \pm 1^{\circ} \mathrm{C}$, que fue controlada diariamente. La aireación fue de 0.5 vvm (15 L.h ${ }^{1}$ ) con aire no enriquecido con $\mathrm{CO}_{2}$. En $\mathrm{pH}$ no fue controlado, pero se verificó diariamente con un pHmetro digital de que se mantuviera dentro del rango óptimo de crecimiento de $N$. oculata $(\mathrm{pH} 7,0$ 9,0). La iluminación recibida fue artificial proveniente de fluorescentes cilíndricos las 24 horas del día, con una iluminancia total de aproximadamente 1000 lux.
Monitoreo del crecimiento

Cada 24 horas se midió con una cámara de Neubauer la densidad celular (células. $\mathrm{mL}^{-1}$ ) de cada uno de los tratamientos. Además se cuantificó diariamente la concentración de clorofila $a\left(\mathrm{mg} \cdot \mathrm{mL}^{-1}\right)$, metabolito primario que funciona como un buen indicador de la biomasa de microalgas (Olvera et al., 2003).

Ensayo de calidad nutricional

En recipientes de $500 \mathrm{~mL}$ se inocularon rotíferos B. plicatilis (30 rotíferos. $\mathrm{mL}^{-1}$ ) y se alimentaron con la microalga $N$. oculata obtenida de los tratamientos (T4 y T6) de la etapa experimental. Como controles se usó $N$. oculata cultivada en medio F/2 (Guillard modificado) y medio Yashima. Se realizó diariamente el conteo de individuos, número de hembras ovadas y número de huevos, utilizando una cámara de Sedgewick-Rafter.

\section{Análisis de datos}

Estimación de los parámetros de crecimiento. Se determinó la tasa de crecimiento del periodo exponencial, ajustándose a una recta la parte lineal de los datos transformados logarítmicamente, $\ln N(t)=a+b t$, en donde $b=\mu_{\exp }$ es la tasa de crecimiento exponencial y $a=\ln N(0)$. El crecimiento de las microalgas se modeló usando la siguiente ecuación logística en tiempo contínuo (Roughgarden, 1998):

$$
N(t)=\frac{K}{1-\left(1-\frac{K}{N_{0}}\right) e^{-\mu_{\max } t}}
$$

Donde $N(t)$ es la densidad celular en el tiempo $t$ (células.mL ${ }^{-1}$ ), $\mu_{\max }$ el tasa máxima de crecimiento $\left(\operatorname{dia}^{-1}\right)$ y $K$ la capacidad de carga del cultivo (células. $\mathrm{mL}^{-1}$ ). Se usó la función logística en tiempo contínuo debido a que la iluminación constante (24 horas al día) elimina el fotoperiodo de las microalgas y determina una división asincrónica (Darley, 1987).

La estimación de los parámetros $\mu_{\max } \mathrm{y} K$ para cada uno de los tratamientos se realizó mediante optimización numérica (método del gradiente conjugado, con derivadas centradas) a fin de minimizar la función de error de cuadrados residuales, usando el programa MATLAB (Mathworks, 2006).

Cálculo de la productividad volumétrica y máximo rendimiento. El modelo de la curva de crecimiento fue normalizado con respecto al tiempo de cultivo a fin de estimar la evolución de la productividad volumétrica $\left(P V\right.$, g. $L^{-1}$. dia $\left.^{-1}\right)$ durante las diferentes fases del cultivo (Crueger \& Crueger, 1989). La fórmula utilizada para la productividad volumétrica se muestra en la ecuación (2).

$$
P V(t)=\frac{1}{t}\left[\frac{K}{1-\left(1-\frac{K}{N_{0}}\right) e^{-\mu_{\max } t}}\right]
$$

Los tiempos que maximizan la PV están determinados por las soluciones de: 


$$
\frac{d P V(t)}{d t}=\frac{N_{t}}{t}\left[-\frac{1}{t}+\mu-\frac{\mu}{K} N_{t}\right]=0
$$

Las soluciones de estas ecuaciones se obtuvieron gráficamente a fin de estimar los tiempos óptimos de producción. El máximo rendimiento $(M R)$ del cultivo representa la máxima cantidad de biomasa que puede extraerse por unidad de tiempo en estado estacionario y está definido por (4):

$$
M R=\frac{\mu_{\max } K}{4}
$$

Estimación de la productividad total y productividad máxima. Se simuló el crecimiento para cultivos de $N$. oculata durante 30 días, considerando un tiempo puesta a punto para los foto-birreactores de de 12 horas. Las curvas de crecimiento poblacional fueron convertidas a biomasa húmeda considerando que $N$. oculata tiene forma esférica, un diámetro aproximado de $4 \mu \mathrm{m}$ y densidad protoplásmica de 1,05 kg.L ${ }^{-1}$ (Tam et al., 2000), obteniéndose una biomasa para $N$. oculata de $2.11 \times 10^{-13} \mathrm{~kg}$.célula ${ }^{-1}$. El cálculo de la productividad total (PT) y productividad máxima (PM) se realizó a partir del modelo obtenido anteriormente y considerando inóculos de $1 \times 10^{6}$ celúlas.mL ${ }^{-1}$.

Estimación de indicadores de producción de rotíferos. Se determinó la tasa de fertilidad (razón entre hembras ovadas y el número total de individuos de la muestra) y el índice de fertilidad (razón entre el número de huevos observados y el total de rotíferos encontrados en la muestra), siendo usados como un indicador cualitativo de la calidad nutricional de la microalga procedente de cada uno de los tratamientos.

Análisis estadístico. Se realizó un análisis de varianza para los parámetros poblaciones, de producción y de calidad nutricional, luego se efectuó la prueba de comparación post-hoc HSD Tukey para determinar las diferencias entre pares de medias de las variables analizadas (Zar, 1999).

Resultados y discusión

Efecto de los medios de cultivos en la dinámica del crecimiento poblacional

Se calcularon las razones atómicas $\mathrm{N}: \mathrm{P}$ para todos los tratamientos en base a la composición química de los medios Guillard F/2, Yashima y los análisis de materia orgánica de los ensilados $\mathrm{H}$ y $\mathrm{E}$ (Tabla 1). Los tratamientos $\mathrm{T} 1$ al $\mathrm{T} 4$ presentaron razones $\mathrm{N}: \mathrm{P}$ de 21:1, 20:1, 25:1 y 23:1 respectivamente (Tabla 2), las cuales se encuentran dentro del rango óptimo (16-
24:1) para las especies de microalgas (Corsini \& Karydis, 1990; Darley, 1987). Los tratamientos T5 y T6 mostraron un exceso en la proporción de nitrógeno, presentando razones N:P de 155:1 y 27:1 respectivamente. Sin embargo, el tratamiento T6 no mostró una limitación debido a que el fósforo se encontraba en cantidades mayores que el tratamiento T1 inclusive (Tabla 3) y el nitrógeno no muestra efectos negativos por exceso de concentración en el medio.

Tabla 1. Composición de los ensilados biológicos de pescado utilizados (hidrolizado y no hidrolizado)

\begin{tabular}{lrr}
\hline & Hidrolizado & No hidrolizado \\
\hline N total $\left(\mathrm{mg} . \mathrm{L}^{-1}\right)$ & 9.49 & 20.00 \\
P total $\left(\mathrm{mg.L}^{-1}\right)$ & 0.31 & 1.68 \\
K total $\left(\mathrm{mg.L} \mathrm{L}^{-1}\right)$ & 3.30 & 6.65 \\
Ca total (mg/L) & 1.67 & 7.36 \\
Mg total (mg/L) & 0.70 & 1.58 \\
Na total (mg/L) & 1.07 & 2.28 \\
B (mg/L) & 30.00 & 12.00 \\
Cu (mg/L) & 2.25 & 2.60 \\
Zn (mg/L) & 4.20 & 7.20 \\
Mn (mg/L) & 2.40 & 2.50 \\
Fe (mg/L) & 21.00 & 64.60 \\
M.O en solución $\left(g . L^{-1}\right)$ & 172.71 & 499.00 \\
\hline
\end{tabular}

Tabla 2. Composición molar de los macronutrientes en cada uno de los tratamientos.

\begin{tabular}{ccccccc}
\hline & T1 & T2 & T3 & T4 & T5 & T6 \\
\hline $\mathbf{N ~ ( m M ) ~}$ & 0.77 & 2.00 & 2.68 & 2.45 & 0.68 & 1.42 \\
$\mathbf{P ~ ( m M ) ~}$ & 0.04 & 0.10 & 0.11 & 0.11 & 0.004 & 0.05 \\
K (mM) & 0.74 & - & 0.07 & 0.05 & 0.07 & 0.18 \\
N:P & $21: 1$ & $20: 1$ & $25: 1$ & $23: 1$ & $155: 1$ & $27: 1$ \\
\hline
\end{tabular}

Tabla 3. Composición química de los medios utilizados en los diferentes tratamientos

\begin{tabular}{rcccccc} 
& T1 & T2 & T3 & T4 & T5 & T6 \\
\hline Macronutrientes & & & & & & \\
N (mg.L $\left.{ }^{-1}\right)$ & 10.76 & 28 & 37.49 & 34.32 & 9.49 & 134.00 \\
P (mg.L $\left.{ }^{-1}\right)$ & 1.12 & 3.15 & 3.29 & 3.24 & 0.14 & 1.68 \\
K (mg/L) & 28.96 & - & 2.73 & 1.82 & 2.73 & 6.90 \\
Ca (mg/L) & - & 4.06 & 5.74 & 5.18 & 1.67 & 7.36 \\
Micronutrientes & & & & & & \\
Mg (mg/L) & - & - & 0.70 & 0.46 & 0.70 & 1.58 \\
Na (mg/L) & 1.42 & - & 1.07 & 0.71 & 1.07 & 2.28 \\
B (mg/L) & - & 0.219 & 0.25 & 0.24 & 0.030 & 0.012 \\
Cu (mg/L) & 0.003 & 0.001 & 0.004 & 0.003 & 0.002 & 0.003 \\
Zn (mg/L) & 0.005 & 0.040 & 0.044 & 0.043 & 0.004 & 0.007 \\
Mn (mg/L) & 0.050 & 0.169 & 0.171 & 0.171 & 0.002 & 0.003 \\
Fe (mg/L) & 0.652 & 0.085 & 0.106 & 0.099 & 0.021 & 0.065 \\
\hline
\end{tabular}

En el tratamiento T1 el nitrógeno se encuentra en forma de nitrato principalmente, a diferencia del 
tratamiento T2 en el que el nitrógeno se encuentra en forma amoniacal y de urea. El nitrógeno en el tratamiento T5 está en forma amoniacal y con aminoácidos libres,

los cuales se encontrarán también disponibles en los tratamientos $\mathrm{T} 2$ y $\mathrm{T} 3$ como resultado del enriquecimiento. En el tratamiento T6 se encontrará nitrógeno amoniacal pero predominará forma proteica

Tabla 4: Parámetros estimados ( \pm límites de confianza al 95 \%) en cada uno de los distintos tratamientos para el cultivo de $N$. oculata.

\section{(péptidos de diferente longitud).}

Se determinó la tasa de crecimiento $\left(\mu_{\text {exp }}\right)$ para los distintos tratamientos (Tabla 4), encontrándose que la mayor tasa de crecimiento en la fase exponencial fue obtenida en el tratamiento T4 (1.13 día $\left.{ }^{-1}\right)$. Esto se explicaría por la utilización del nitrógeno amoniacal y la presencia de aminoácidos, que son de más fácil asimilación por la microalga, más que por las diferencias en la razón $\mathrm{N}: \mathrm{P}$ con los otros tratamientos. No se observaron diferencias significativas $(p>0,05)$ entre T1 (0.98 día $\left.{ }^{-1}\right)$, T2 (0.95 día $\left.{ }^{-1}\right)$, T3 (0.98 día $\left.{ }^{-1}\right)$ y T6 (0.88 día $\left.{ }^{-1}\right)$ (Tabla 6). Esto se explicaría por la mayor concentración de enriquecimiento del tratamiento 3, a diferencia de T4, pudo haber producido una represión catabólica pues a concentraciones de amonio mayores a 0.5 moles. $\mathrm{L}^{-1}$ las microalgas dejan de asimilar nitrógeno en forma de nitrato o urea (Darley, 1987), y con ello el enriquecimiento y el incremento de la razón $\mathrm{N}: \mathrm{P}$ no produjeron la mejora observada en el tratamiento 4 . Así, los resultados de los tratamientos T1, T2 y T3, con razones $\mathrm{N}: \mathrm{P}$ similares, no mostraron diferencias significativas. El tratamiento T6, a pesar de no tener una razón N:P óptima, contenía nutrientes en cantidad suficiente como para un adecuado desarrollo de $N$. oculata, en cantidades por encima inclusive que el control (T1) por lo que los resultados no difirieron significativamente de los observados en los tratamientos T1, T2 y T3. El T5 mostró la menor tasa de crecimiento (0.65 día $\left.{ }^{-1}\right)$, debido al sesgo de la pronta aparición del efecto de la denso-dependencia, debido al rápido agotamiento de los nutrientes, como se observará más adelante al analizar la capacidad de carga de cada uno de los tratamientos. Las tasas de crecimientos durante la fase exponencial fueron mayores que la estimada en el T7 a partir de los datos de Chinchayán (1996) para el cultivo de $N$. oculata en harina de pescado, con un valor máximo de 0.81 día $^{-1}$. La tasa de crecimiento durante la fase exponencial del cultivo es un indicador utilizado comúnmente en los trabajos de acuicultura, lo que nos permite una comparación de los resultados con otros trabajos, aunque para los fines de analizar el rendimiento y la productividad de un cultivo no es suficiente y tendrán que analizarse los parámetros durante toda la curva de crecimiento. 
posibilitó una mayor disponibilidad de metales para el desarrollo del cultivo. Por otro lado, el T6, a pesar de no tener una relación N:P óptima, en términos absolutos podía suministrar suficientes macro $\mathrm{y}$ micronutrientes para el adecuado desarrollo de $N$. oculata. En segundo lugar, no se observaron diferencias significativas entre los tratamientos T4 $\left(602.44 \times 10^{5}\right.$ células.mL $\left.{ }^{-1}\right)$ y T3 $\left(507,03 \times 10^{5}\right.$ células . $\left.\mathrm{mL}^{-1}\right)$. Estos resultados son aparentemente contradictorios pues los tratamientos T3 y T4 contenían más macro y micronutrientes en términos absolutos en el tratamiento T2 (medio Yashima), sin embargo, presentaron menores valores de capacidad de carga. Esto se debe a que la disponibilidad de $\mathrm{CO}_{2}$ disuelto en el agua será el principal determinante de la capacidad de carga si algún otro nutriente soluble no es limitante. En el medio, el $\mathrm{pH}$ afecta la solubilidad del $\mathrm{CO}_{2}$, disminuyendo esta drásticamente en medios alcalinos. Se observó que los máximos valores de $\mathrm{pH}$ observados (9.0) se registraron en los tratamientos T3 y T4. Además, se puede observar que el incremento de $\mathrm{pH}$ hasta 9.0 es abrupto, en especial en el T3 (Figura 1), lo que es un indicador
que el carbono era limitante en el medio (Harrison \& que el carbono era limitante en el medio (Harrison \& Berges, 2004). En tercer lugar estuvo el T1 (260.15 $\times$ $10^{5}$ células. $\mathrm{mL}^{-1}$ ), del cual notamos que a pesar de estar diseñado para satisfacer todos los requerimientos nutricionales de la microalga, las concentraciones absolutas de los macronutrientes están en cantidades mucho menores que la del medio Yashima, por ejemplo. Esto se deriva en que, a largo plazo, el T1 no pueda soportar densidades celulares muy altas. Finalmente, el T5 presentó una capacidad de carga de $40.48 \times 10^{5}$ céls. $\mathrm{mL}^{-1}$, valor que se encuentra en un orden de magnitud por debajo de los demás. Así, a pesar de que aparentemente $\quad N$. oculata no tuvo problemas para su asimilación y presentó efectos positivos en el enriquecimiento, los

Tabla 6: Resultados de la prueba de comparación post-hoc HSD Tukey para los parámetros de producción (Subconjuntos homogéneos para $\alpha=0.05$ ).

\begin{tabular}{cccccccccc} 
Trat & N & \multicolumn{3}{c}{ Máximo rendimiento } & \multicolumn{5}{c}{ Productividad volumétrica } \\
Sub-conjunto & 2 & 3 & 4 & 1 & 2 & 3 & 4 & 1 \\
\hline 5 & 3 & 0.20 & & & & 0.18 & & & \\
1 & 3 & & 1.63 & & & & 0.97 & & \\
3 & 3 & & 2.31 & 2.31 & & & 1.24 & 1.24 & \\
6 & 3 & & & 2.85 & 2.85 & & & 1.69 & 1.69 \\
4 & 3 & & & 3.19 & 3.19 & & & & 1.54 \\
2 & 3 & & & & 3.50 & & & & 1.81 \\
Sig. & & 1.000 & .181 & .056 & .210 & 1.000 & .263 & .173 & .257 \\
\hline
\end{tabular}

nutrientes se encontraban en concentraciones muy bajas, por lo que no pudo soportar concentraciones más altas de células, llegando a la saturación a los pocos días del cultivo. Salvo por el T5, las capacidades de carga observadas fueron mayores que las estimadas para el T7 de los datos de Chinchayán (1996) para el cultivo de $N$. oculata en harina de pescado, con un valor máximo de $199.40 \times 10^{5}$ células. $\mathrm{mL}^{-1}$.
Máximos rendimientos y productividad volumétrica

Se estimaron los máximos rendimientos $(M R)$ para fue obtenido por el T2 (3.50 g.L L $^{-1}$.día ${ }^{-1}$ ), el T4 (3.19 g. $L^{-1}$.día $\left.{ }^{-1}\right)$ y el T6 (2.85 g. $L^{-1}$.día $\left.{ }^{-1}\right)$, entre los cuales no se encontraron diferencias significativas $(p>0,05)$ (Tabla 6). Luego se encontraron los tratamientos el T3 (2.31 g. L $^{-1}$.día $\left.{ }^{-1}\right)$ y el T1 (1.63 g.L L $^{-1}$.día $\left.{ }^{-1}\right)$, seguidos de T5 (0.2 g. $\mathrm{L}^{-1}$.día $\left.{ }^{-1}\right)$. La comparación efectuada con los resultados del T7 sobre harina de anchoveta (Chinchayán, 1996) muestra que el enriquecimiento orgánico (T3 y T4) y el cultivo en medios orgánicos (T6) mostraron mejores rendimientos que los obtenidos con la harina ( 0.75 g.L $\mathrm{L}^{-1}$.día $\left.{ }^{-1}\right)$, el cual es un producto de mayor valor económico que los residuos $N$. oculata (Tabla 5). El mayor máximo rendimiento

Tabla 5: Parámetros ( \pm límites de confianza al $95 \%$ ) asociados a la producción del cultivo

\begin{tabular}{lccc}
\multicolumn{1}{c}{ de $N$. oculata en cada uno de los distintos tratamientos. } \\
\multicolumn{1}{c}{ Tratamiento } & $\begin{array}{c}\boldsymbol{M R} \\
\text { (g.L } \mathbf{L}^{-1}\end{array}$ & $\begin{array}{c}\boldsymbol{P V}_{\max } \\
\text { (g.L }^{-1} \text { ) }\end{array}$ & $\begin{array}{c}\mathbf{t}_{\max } \\
\text { (días) }\end{array}$ \\
\hline T1. Guillard F/2 & $1.63 \pm 0.44$ & $0.97 \pm 0.20$ & 5.00 \\
T2. Yashima & $3.50 \pm 0.87$ & $1.81 \pm 0.28$ & 8.00 \\
T3. Yashima + ensilado-H 1/1000 & $2.31 \pm 0.87$ & $1.24 \pm 0.44$ & 7.33 \\
T4. Yashima + ensilado-H 1/1500 & $3.19 \pm 0.87$ & $1.54 \pm 0.42$ & 7.00 \\
T5.Ensilado-H 1/1000 & $0.20 \pm 0.13$ & $0.18 \pm 0.08$ & $<4.00$ \\
T6.Ensilado-E 1/1000 & $2.85 \pm 1.02$ & $1.69 \pm 0.42$ & 6.67 \\
T7.Harina de anchoveta (Chinchayán 1996) & 0.75 & 0.39 & 9.00
\end{tabular}

con los que se fabrica el ensilado.

Se calculó la productividad volumétrica para todo el tiempo de cultivo en lote, estimándose la productividad máxima para cada uno de los tratamientos y el tiempo necesario para alcanzar tal productividad máxima (Tabla 5). No se encontraron diferencias significativas en la productividad volumétrica entre los tratamientos T2 (1.81 g. $\mathrm{L}^{-1}$.día $\left.{ }^{1}\right)$, T6 (1.69 g.L ${ }^{-1}$.día $\left.{ }^{-1}\right)$ y T4 (1.54 g.L . día $\left.^{-1}\right)$, sin embargo, T4 tampoco mostró diferencias con T3 (1.24 g. $\mathrm{L}^{-1}$. día $\left.{ }^{-1}\right)$, por lo que consideramos que T2 y T6 mostraron los mejores resultados (Figura 2, Tabla 6).

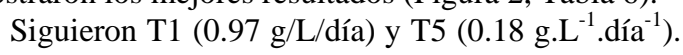
La productividad volumétrica estimada para los datos de Chinchayán (1996) fue menor que todos los tratamientos (0.39 g. $\mathrm{L}^{-1}$. día $\left.^{-1}\right)$, salvo el T5, lo que indica que el cultivo de $N$. oculata en ensilado biológico de pescado proporcionaría un mejor rendimiento y producción que sobre harina de anchoveta. Los tiempos necesarios para alcanzar la productividad máxima fueron estimados a partir del 
método gráfico y mediante aproximaciones numéricas (ecuación 5). El menor tiempo fue inferior a 4 días (T5), seguido del T1 con 4 días. En el caso del T5, la estimación no fue del todo confiable debido a que la capacidad de carga se alcanzó muy rápidamente, antes del quinto día inclusive. No se observaron diferencias significativas $(p>0,05)$ entre el T6 con 6.7 días y el T4 con 7.0 días. A estos les siguieron el T3 con 7.3 días y el T2 con 8,0 días. Se estimó también la productividad total, considerando un tiempo de puesta a punto entre cosecha y cosecha de 12 horas (Figura 3).

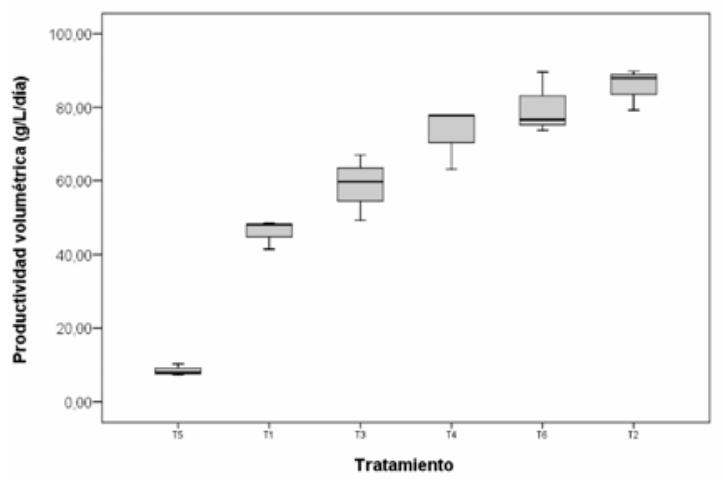

Figura 2: Productividad volumétrica (PV) de la microalga N. oculata (g.L-1.mes-1) de cultivo para los diferentes tratamientos.

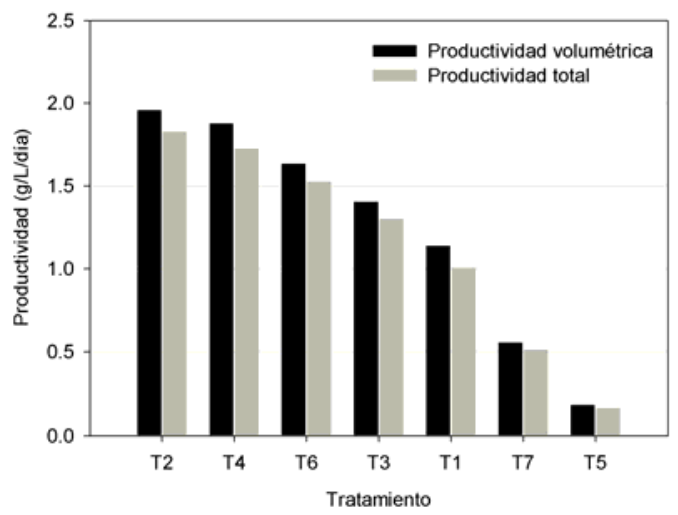

Figura 3: Comparación de la máxima productividad volumétrica (PV) y la productividad total (PT) de los diferentes tratamientos del cultivo de $N$. oculata, para

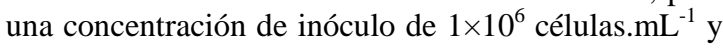
un tiempo de puesta a punto de 12 horas.

Evaluación de la calidad nutricional

Se utilizaron los datos de índice de fertilidad y tasa de reproducción de todos los tratamientos para determinar cual sería el mejor momento para comparar los resultados de los diversos tratamientos. Se compararon los resultados del día 2 por ser el más representativo al presentar los mayores valores y el día que se registró la mayor dispersión.
En la Figura 4 se comparan los resultados de la tasa de reproducción para los tratamientos T1, T2, T4, y T6 durante el día dos, se muestra la desviación estándar de las distintas repeticiones. Los mejores resultados fueron obtenidos por el T2, seguido del tratamiento T1, T6 y T4.

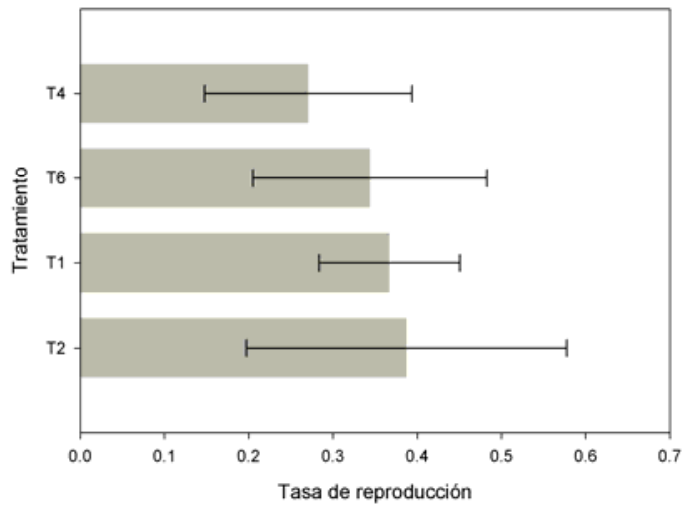

Figura 4: Tasa de reproducción (hembras ovadas/rotífero) del cultivo de $B$. plicatilis en el segundo día de cultivo, alimentados con algas de cuatro de tratamientos.

Los índices de fertilidad se muestran en la Figura 5 en donde se observa que el T6 presentó el mayor índice, asociado con una buena producción de huevos e indicador de una buena calidad nutricional de las microalgas obtenidas en ese tratamiento. Esto pudo deberse a que $N$. oculata cultivada mixotroficamente aumenta la producción de ácidos grasos poliinsaturados (Fang et al., 2004) necesarios para la reproducción de Brachionus plicatilis. Este resultado fue seguido por el T1, Guillard modificado F/2, que es un medio estandarizado para satisfacer todas las necesidades nutricionales de las microalgas, lo que asegura que estas sean, a su vez, un buen alimento para B. plicatilis. Luego siguieron los tratamientos T4 y $\mathrm{T} 2$ respectivamente, que no presentaron diferencias significativas.

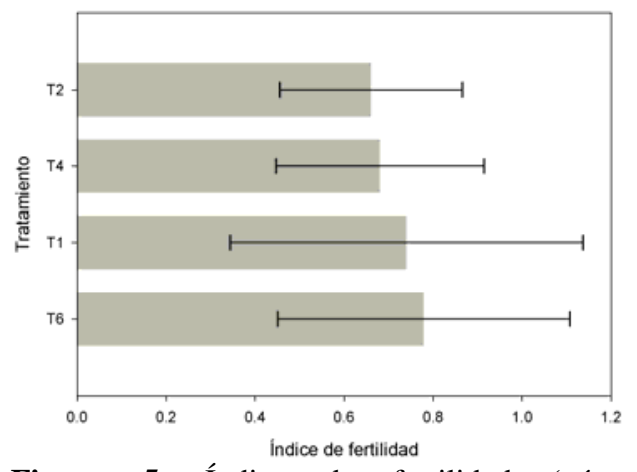

Figura 5: Índice de fertilidad (número de huevos/rotífero) de $B$. plicatilis en el segundo día de cultivo, alimentado con algas de cuatro tratamientos. 
Simulación de la producción total mensual

A fin de determinar bajo qué tratamientos podría obtenerse la mayor producción total de microalga, se tomó como referencia un mes (30 días) para la producción y se simuló el cultivo utilizando los diferentes tratamientos. La cosecha se realizó cuando el cultivo alcanzaba su productividad máxima y se consideró un tiempo de puesta a punto de 12 horas.

Los resultados muestran que los volúmenes de producción con el T6 y el T4 son similares a los obtenidos utilizando el medio Yashima (T2), ampliamente utilizado en producción acuícola (Figura $6)$.

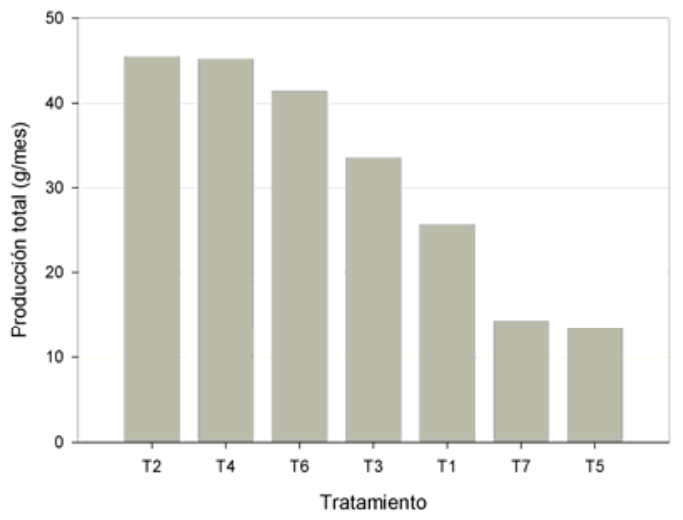

Figura 6: Producción mensual de la microalga $N$. oculata (g. $\mathrm{L}^{-1} \cdot \mathrm{mes}^{-1}$ ) de cultivo para los diferentes tratamientos.

Se puede observar aquí el efecto combinado del tiempo y la productividad máxima, pues el $\mathrm{T} 4$ se colocó por encima del T6 que presentó una mayor productividad volumétrica. Sin embargo, estos resultados están basados en los promedios de los parámetros determinados, para los cuales no se encontraron diferencias significativas, por lo que la producción con los medios de los tratamientos T4, T2 y T6 deberían ser esencialmente equiparables. Así mismo, si bien el enriquecimiento con ensilado de pescado (T4) no produce mejoras significativas en la producción, si lo hace en la tasa de crecimiento máxima y el tiempo óptimo de producción, como se vio anteriormente. Sin embargo, la adición de ensilado al medio Yashima aumentaría innecesariamente los costos de producción sin mejorar la productividad. Por lo tanto, en términos del costo total no sería rentable enriquecer los cultivos con ensilado si ya se está utilizando un medio químico como el Yashima, pues tampoco se observaron mejoras en la calidad nutricional que justifiquen el gasto adicional. Por otra parte, el T6 mostró una mejor calidad nutricional como alimento vivo para $B$. plicatilis lo que sería una ventaja adicional, pues los costos del medio con ensilado (S/. 2.50 el litro) son similares al del Yashima (S/. 2.20 el litro) basado en insumos agrícolas.
Esta ventaja es muy deseable a la vez, dado que $N$. oculata es cultivada como alimento vivo, y en última instancia nos interesará su calidad como alimento más que su volumen bruto de producción.

Para posteriores estudios se sugiere buscar una fuente alternativa de fósforo para mejorar el balance $\mathrm{N}: \mathrm{P}$ del ensilado biológico de pescado y evaluar su efecto en la producción. Además, evaluar la posibilidad de usar el ensilado biológico de pescado como sustrato para crecimiento heterotrófico de microalgas y la calidad nutricional de la microalga en otros consumidores (Artemia, copépodos, pulgas de agua, etc.).

\section{Conclusiones}

La producción volumétrica, que es el mejor criterio de selección de tratamientos, mostró que el ensilado biológico de pescado no-hidrolizado (T6) fue tan bueno como los medios T2 (Yashima) y T4 (Yashima + ensilado-H 1/1500).

El efecto combinado de medios inorgánicos con ensilado de pescado (T4, Yashima + ensilado-H 1/1500) no mejora significativamente la producción ni la calidad nutricional de $N$. oculata.

El ensilado biológico de pescado no-hidrolizado (T6) presentó el mayor índice de fertilidad para el rotífero B. plicatilis, indicador de una buena calidad nutricional de las microalgas.

El ensilado biológico de pescado tiene un efecto positivo sobre el crecimiento de la microalga $N$. oculata, lo que indica que puede suministrar los nutrientes necesarios para su adecuado desarrollo.

El ensilado biológico de pescado puede ser usado como medio de producción masiva para la microalga N. oculata.

\section{Agradecimientos}

Agradecemos al biólogo Jorge Bautista del Laboratorio de Acuicultura del Instituto del Mar del Perú por la donación de la cepa de Nannochloropsis oculata. Al técnico Eberth Vicente Armas y a los asistentes de investigación del Laboratorio de Biorremediación de la UNALM por el apoyo en el trabajo de laboratorio. Al Dr. Leoncio Ruiz del departamento de Acuicultura de la UNALM por facilitar las instalaciones del Laboratorio de Acuicultura para ejecutar el experimento. Agradecemos también al Dr. Jorge Tam por sus valiosas sugerencias en la discusión de los resultados y a los profesores Dra. Patricia Gil Kodaka, Dr. Víctor Meza y M. Sc. Alfredo Rodríguez por sus sugerencias y críticas al borrador de este trabajo en su versión de tesis de grado. Finalmente, agradecemos a dos revisores anónimos por sus acertadas sugerencias que ayudaron a mejorar este trabajo. 


\section{Literatura citada}

Brown M., Mular M., Miller I., Farmer C. \& Trenerry C. 1999. The vitamin content of microalgae used in aquaculture. Journal of Applied Phycology, 11: 247-255.

Chinchayán M. 1996. Cultivo de la microalga Nannochloropsis oculata y su consumo por el rotífero Brachionus plicalitis (línea S). Tesis (Biólogo). Lima, Perú. Universidad Nacional Agraria La Molina, Facultad de Ciencias. 104 p.

Corsini M. \& Karydis M. 1990. An algal medium based on fertilizers and its evaluation in mariculture. Journal of Applied Phycology 2: 333-339.

Crueger W. \& Crueger A. 1989. Biotecnología: Manual de Microbiología Industrial. Tercera edición. España, Editorial Acribia. 413 p.

Darley W. 1987. Biología de las Algas. Enfoque Fisiológico. México, Editorial Limusa. 236 p.

De la Cruz S. A. \& Alfonso E. 1975. Cultivo masivo de algas planctónicas marinas mediante fertilización. Ciencias, 8:1-25.

Fang X., Han-Hua H., Wei C., Zhao-Ling C. \& Fan O. 2004. Growth characteristics and eicosapentaenoic acid production by Nannochloropsis sp. in mixotrophic conditions. Biotechnology Letters 26: 51-53.

González D. \& Marín M. 2005. Obtención de ensilados biológicos a partir de los desechos del procesamiento de sardinas. Revista Científica, FCV-LUZ 15(6): 560-567.

Granados C. \& Bückle L. 1983. Cultivo de las microalgas Monochrysis lutheri y Skeletonema costatum con nutrientes producidos por estiércoles digeridos. Anales del Instituto de Ciencias del Mar y Limnología 11 (1): 241-256.

Harrison P. \& Berges J. 2004. Marine Culture Media. AMCE Program, Hong Kong University of Science and Technology Department of Biological Sciences, University of Wisconsin-Milwaukee, 3: 21-35

Hoshida H., Ohira T., Minematsu A., Akada R. \& Nishizawa Y. 2005. Accumulation of eicosapentaenoic acid in Nannochloropsis sp. in response to elevated CO2 concentrations. Journal of Applied Phycology. 17: 2934.
Leal S., Delgado G. \& Almaguer Y. 2004. Efecto de cinco tipos de productos zeolíticos sobre el crecimiento de la microalga Nannochloropsis gaditana. Revista de Investigaciones Marina, 25(3): 241-244.

Mathworks. 2006. MATLAB The language for technical computing: reference manual. Maryland, EUA. The Mathworks, Inc. 874p

Olvera R., Ríos E. \& Vicente V. 2003. Manual de técnicas para el cultivo y extracción de Bioproductos a partir de Microalgas. México. Escuela Nacional de Ciencias Biológicas (ENCB). Instituto Politécnico Nacional (IPN). $70 \mathrm{p}$.

Otero A., García D. \& Fabregas J. 1997. Factors controlling eico-sapentaenoic acid production in semicontinuous cultures of marine microalgae. Journal of Applied Phycology, 9: 465-469.

Paniagua J. \& Bückle L.1983. Cultivo en condiciones controladas de Monochrysis lutheri y Skeletonema costatum con extractos de macrofitas marinas (fitoplancton). Anales del Instituto de Ciencias del Mar y Limnología. Universidad Nacional Autónoma de México, 12(1): 59-70.

Roughgarden J. 1998. Primer of ecological theory. New Yersey, EUA. Editorial Prentice-Hall. 456p.

Sukenik A., Zmorab O. \& Carmeli Y. 1993. Biochemical quality of marine unicellular algae with special emphasis on lipid composition. II. Nannochloropsis sp. Aquaculture, 117: 313-326

Tam J., Vera G., Pinto E. \& Melgar R. 2000. Modelo de simulación de los efectos ecotoxicologicos del cadmio sobre el crecimiento poblacional de la microalga Skeletonema costatum (Greville) Cleve. Inf. Prog. Inst. Mar Perú, 130:3-12.

Veral A., Martínez M., Morillo K. \& Montes S. 2006. Cultivo discontinuo de Chlorella sp. en medios enriquecidos con el exudado gomoso de Acacia macracantha. Boletín del Centro de Investigaciones Biológicas, 38 (2): 109-11.

Zar J.H. 1999. Biostatistical Analysis. 4th Edition. New Jersey, Editorial Prentice-Hall, 931 pp. 


\section{Figura 1 citada en el texto}
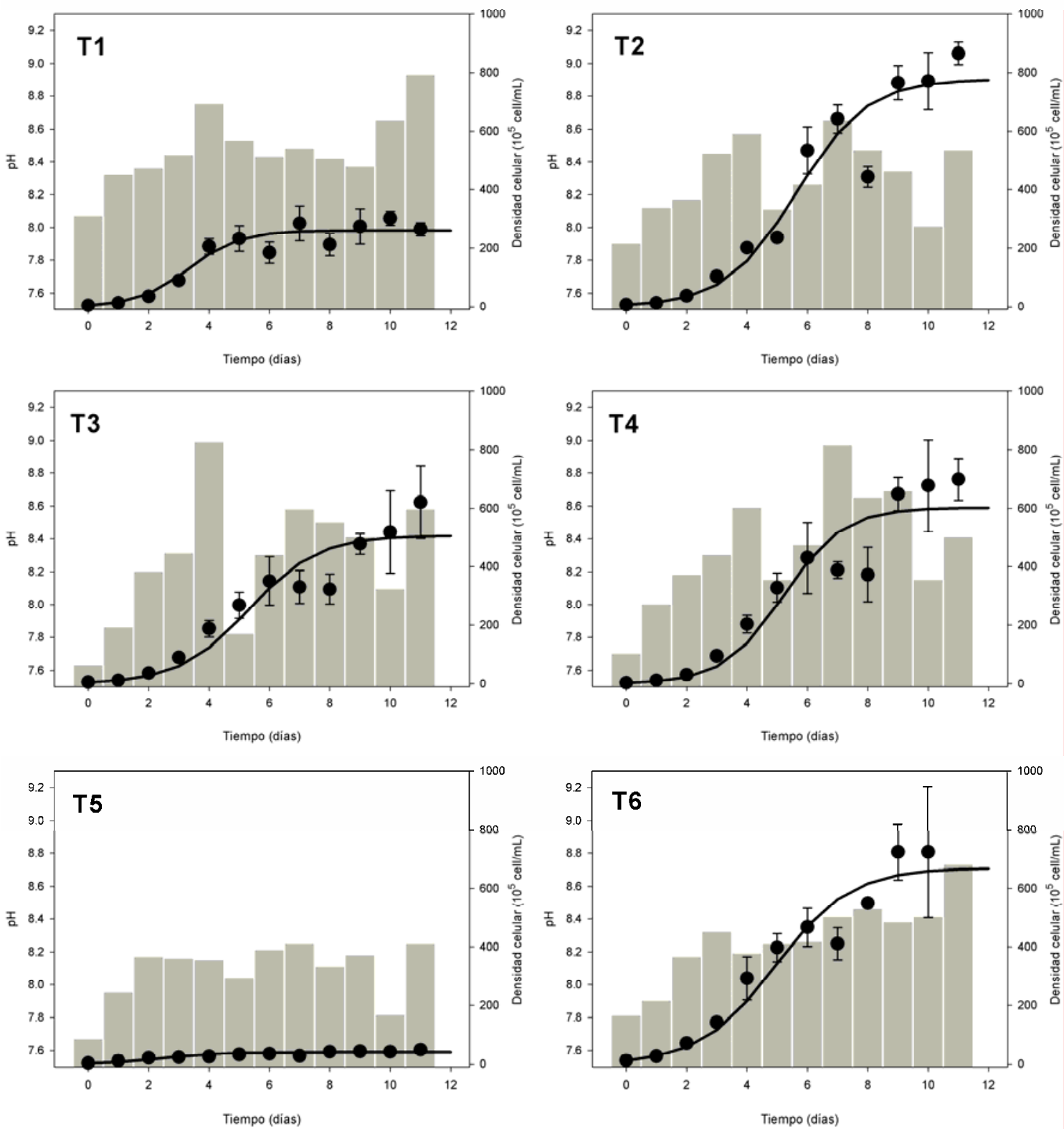

Figura 1: Curvas de crecimiento de $N$. oculata (datos simulados en líneas, datos observados en puntos, $\mathrm{n}=3$ ) y evolución del pH (barras) durante el cultivo.

${ }^{1}$ Laboratorio de Biorremediación. Facultad de Ciencias, Universidad Nacional Agraria La Molina. Av. La Molina s/n, Lima 12, Perú. heidi.isabel@gmail.com,jjmorales_2005@yahoo.es

${ }^{2}$ Laboratorio de Acuicultura. Facultad de Pesquería, Universidad Nacional Agraria La Molina. Av. La Molina s/n, Lima 12, Perú. jesvargas@lamolina.edu.pe

${ }^{3}$ Departamento de Ciencias, Pontificia Universidad Católica del Perú. Av. Universitaria 1801, San Miguel, Lima 32, Perú. ricardo.oliveros@pucp.edu.pe 